\title{
Lösungsmöglichkeiten nach fehlgeschlagenen Osteosynthesen: Calcaneus
}

\author{
Hans Zwipp, Stefan Rammelt, René Grass
}

\section{Zusammenfassung}

Fehlgeschlagene Osteosynthesen am Calcaneus sind in den meisten Fällen durch eine unzureichende anatomische Rekonstruktion, besonders der subtalaren Gelenkfacette, bedingt. Nur gelegentlich kommt es durch die Wahl eines falschen Zugangs, des ungeeigneten Implantates, mangelhafter Fixationstechnik oder am seltensten durch die fehlende Compliance des
Patienten in der Nachbehandlung zu Fehlschlägen. Lösungsmöglichkeiten zur Frühkorrektur von fehlgeschlagenen Osteosynthesen am Calcaneus, insbesondere mit dem Ziel der Gelenkerhaltung innerhalb der ersten 3 bis 6 Wochen postoperativ sind sehr begrenzt. Dies hat mehrere Gründe wie die rasche Fehlverheilung, die postoperative Inaktivitätsosteoporose und das erhöhte Infektrisiko.

\section{Rasche Fehlverheilung:}

Der vornehmlich spongiöse Calcaneus heilt rasch knöchern, d.h. nach ca. 3 Wochen ist eine fehlgestellte Fraktur durch Kallus meist so fest, dass nur durch einoder mehrdimensionale Osteotomie eine Korrektur möglich erscheint.

\section{Postoperative Inaktivitätsosteoporose:}

Durch den postoperativen Nicht- oder Mindergebrauch des Fußes setzt eine rasche Inaktivitätsosteoporose des Calcaneus ein, so dass Korrekturoperationen mit mangelhaftem Halt der Schrauben in dieser Phase extrem schwierig sind, insbesondere wenn kein winkelstabiles Implantat zur Verfügung steht.

\section{Weichteil- und Infektrisiko}

Durch die primär einwirkende Traumaenergie und durch das notwendige operative Trauma zur offenen Versorgung einer Calcaneusfraktur ist die Weichteilsituation bei ohnehin schlechter Weichteilummantelung des Calcaneus in den ersten postoperativen Wochen i.d.R. so prekär, dass ein erheblich gesteigertes

OP-JOURNAL 2004; 20: 236-242

(c) Georg Thieme Verlag KG Stuttgart · New York

Risiko für einen erzwungenen Wunderverschluss und eine Weichteil- und/ oder Knocheninfektion bei frühen Revisionseingriffen gegeben ist.

\section{Lösungsmöglichkeiten zur Spätkorrek-} tur, d.h. ca. 6 bis 12 Monate postoperativ, können die Gesamtsituation bei minimierten Risiken deutlich verbessern, da die Knochendichte wieder normalisiert ist und die Weichteile sich erholt haben. Sie implizieren aber, dass eine anatomische Rekonstruktion der Gelenkabschnitte nicht mehr möglich ist, weswegen nur noch die Option einer reorientierenden subtalaren Arthrodese verbleibt.

Neue Möglichkeiten zur Frühkorrektur haben sich durch neue bildgebende Technik wie ISO C 3D [4], intraoperative Arthroskopie [2,3] und innovative Implantate wie die der winkelstabilen Calcaneusplatte [5] ergeben, was in der vorliegenden Arbeit aufgezeigt werden soll.

\section{Vermeidung von Fehlschlägen}

Während eine ungenügende Aufrichtung des Fersenbeines mit einem Böhler-Winkel erst ab einer Differenz von 30\% und mehr in Relation zur gesunden Seite zu signifikant schlechteren Ergebnissen führt, sind bereits minimale Stufen in der posterioren Facette von 1 bis $2 \mathrm{~mm}$ signifikant schlechter im funktionellen
Resultat [3]. Deshalb müssen zur Vermeidung einer unzureichenden Osteosynthese alle intraoperativen diagnostischen Kontrollmöglichkeiten ausgenutzt werden, um keine, noch so kleine, Gelenkstufe zurückzulassen. Dies mit dem Ziel sofort, d.h. noch intraoperativ nachkorrigieren zu können, bevor die Fehlstellung durch Schrauben und Platte fixiert wird. Weder das bloße Auge, noch die BVoder intraoperative Röntgenkontrolle, selbst die 20 Grad Brodén-Aufnahme, ist so zuverlässig wie die offene arthroskopische Visualisation unter Varusstress des Fersenbeines bei liegender SchanzSchraube im Tuber calcanei (Abb.1ad). So konnte im eigenen Vorgehen in 59 kontrollierten offenen Osteosynthesen des Fersenbeines unter arthroskopischer Kontrolle der posterioren Facette in 13 Fällen (22\%) überraschend eine Gelenkstufe von 1 bis $2 \mathrm{~mm}$ erkannt und anschließend sofort korrigiert werden [2].

Ist eine intraoperative arthroskopische Kontrolle nicht möglich und steht ein ISO C 3D-Bildwandler nicht zur Verfügung, sollte bei intraoperativ nicht sicher beurteilbaren Brodén-Aufnahmen und möglichst zu verwendenden Titanimplantaten ein postoperatives CT zur Beurteilung der Gelenkfacetten veranlasst werden, um gegebenenfalls so rasch wie möglich nachzukorrigieren.

Bei diesem grundsätzlichen Vorgehen mit postoperativer CT-Kontrolle sollte der Patient allerdings im präoperativen Aufklärungsgespräch bereits darauf hingewiesen werden, dass das postoperative CT zur Qualitätskontrolle dient, damit auch kleinere verbliebene Gelenkstufen einer Korrektur früh zugeführt werden können.

\section{Frühkorrektur (innerhalb 3 bis 6 Wochen postoperativ)}

a) Fehlstellung

Frühe Korrekturen einer Osteosynthese am Fersenbein, d.h. innerhalb von 3 bis 6 Wochen postoperativ sind nur selten 


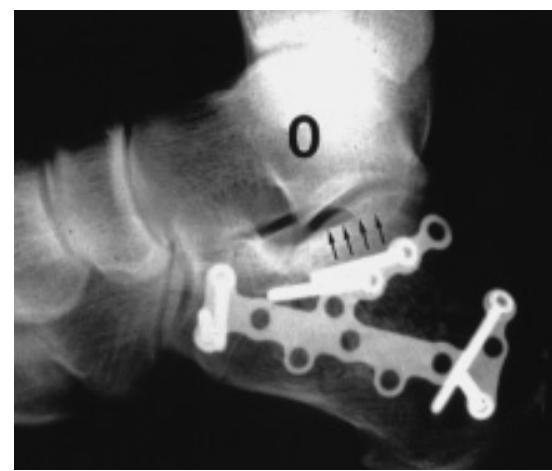

b
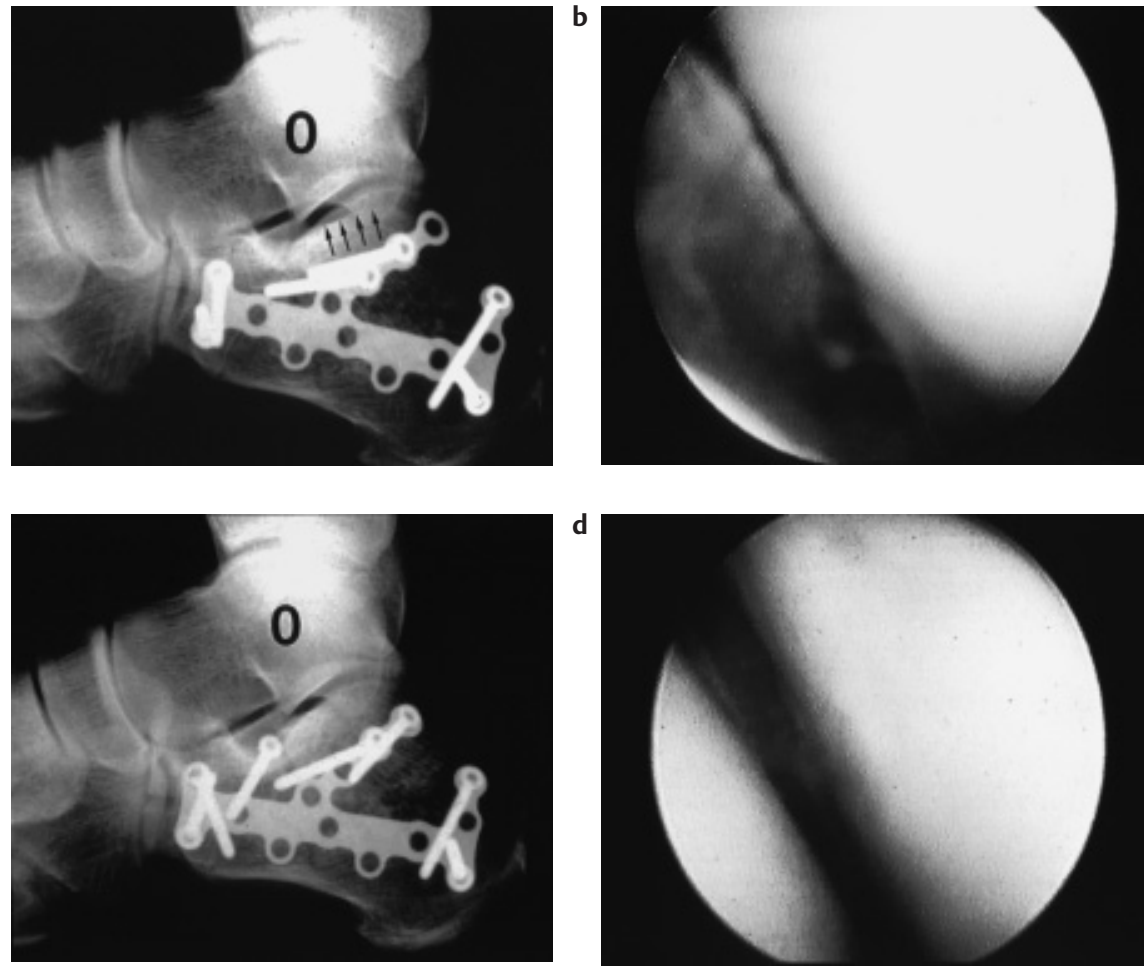

Abb.1 Intraoperative offene Arthroskopie des Subtalar-Gelenkes. (a) Scheinbar gutes Rekonstruktionsergebnis bei bereits liegender Sanders-Platte. Es besteht eine unklare Doppelkontur der posterioren Facette (schwarze Pfeile). (b) Die intraoperative offene Arthroskopie unter Varusstress des Rückfußes lässt eine deutliche Stufe der posterioren Facette von mindestens 2 mm erkennen. (c) Nach Nachkorrektur und Neuplatzierung der beiden subthalamischen Schrauben erscheint das Röntgenbild jetzt im Vergleich zu (a) korrekt, was sich in der nachkontrollierenden arthroskopischen Visualisation (d) als stufenlose Reposition sicher nachweisen lässt. möglich, da sie technisch extrem schwierig und insgesamt sehr risikoreich sind. Dies ist darin begründet, dass einerseits die Knochenheilung am spongiösen Knochen rasch fortschreitet, die Weichteile verbacken, die Haut vulnerabel ist und andererseits notwendige Osteotomien, ein weicher, inaktivitätsosteoporotischer Knochen die Osteosynthesetechnik erheblich erschweren, so dass am Ende einer langwierigen Reoperation die hohe Gefahr des nicht primär erzielbaren Wundverschlusses besteht, was zusätzlich eine Weichteil-Knocheninfektion grundsätzlich begünstigt. Im klinischen Fallbeispiel 1 (Abb.2) soll gezeigt werden, dass der Patient über die hohen Risiken einer solchen Frühkorrektur lückenlos aufgeklärt werden muss und dass die Option einer alternativen Subtalar-Arthrodese bei Nichtrekonstruierbarkeit der posterioren Facette vom Patienten bewilligt sein sollte. Dies mit dem Hinweis, dass bei einer Spätkorrektur (nach 6 bis 12 Monaten) das hohe Risiko der Frühkorrektur entfällt bzw. minimiert wird, dann jedoch eine einsteifende, reorientierende Subtalar-Arthrodese obligat wird.
Abb. 2

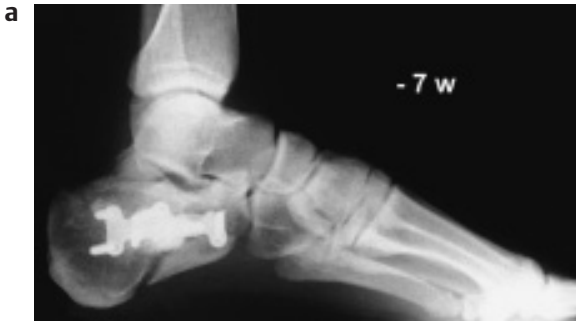

b

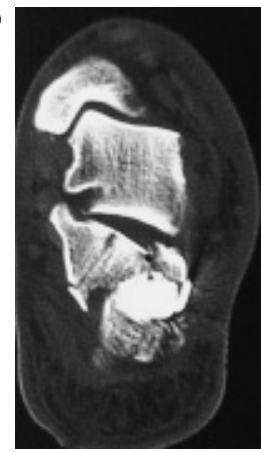

d

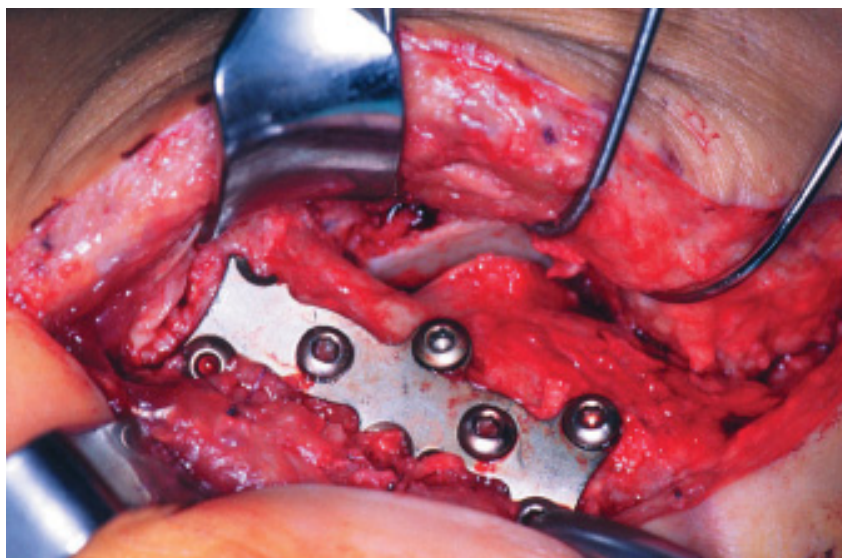

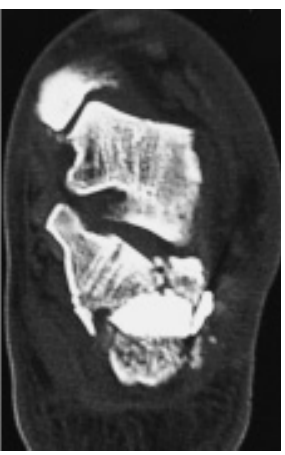
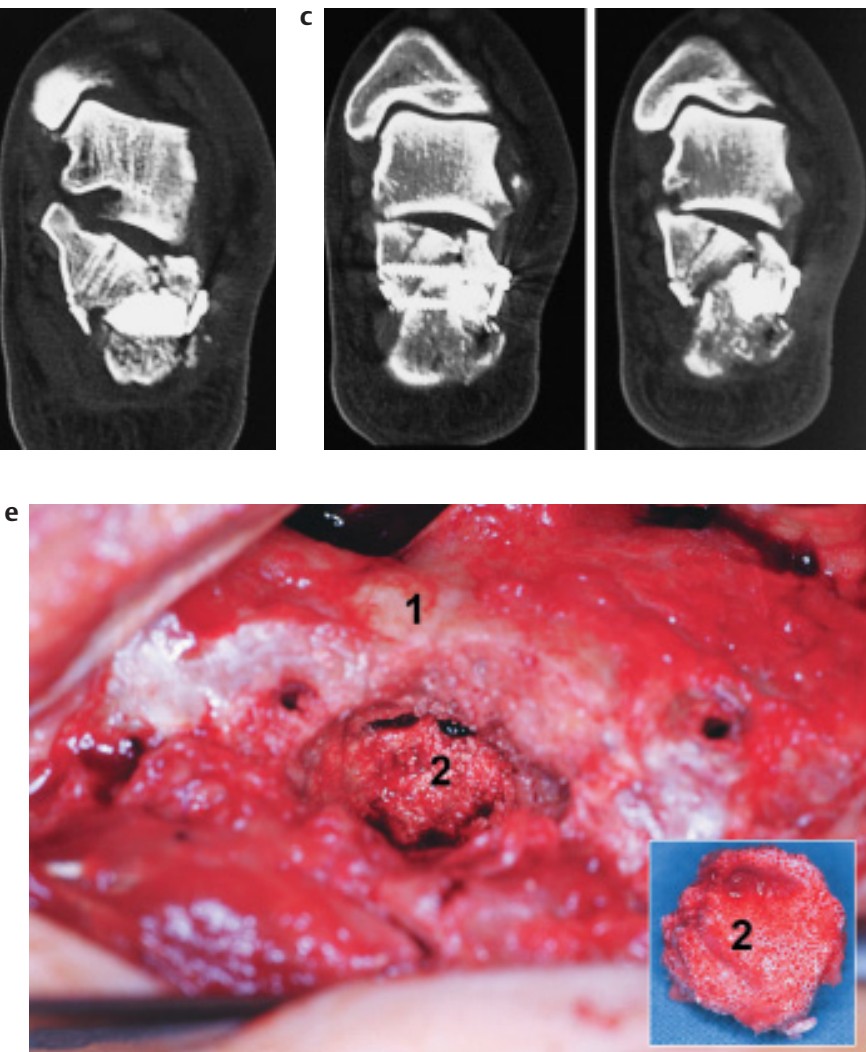

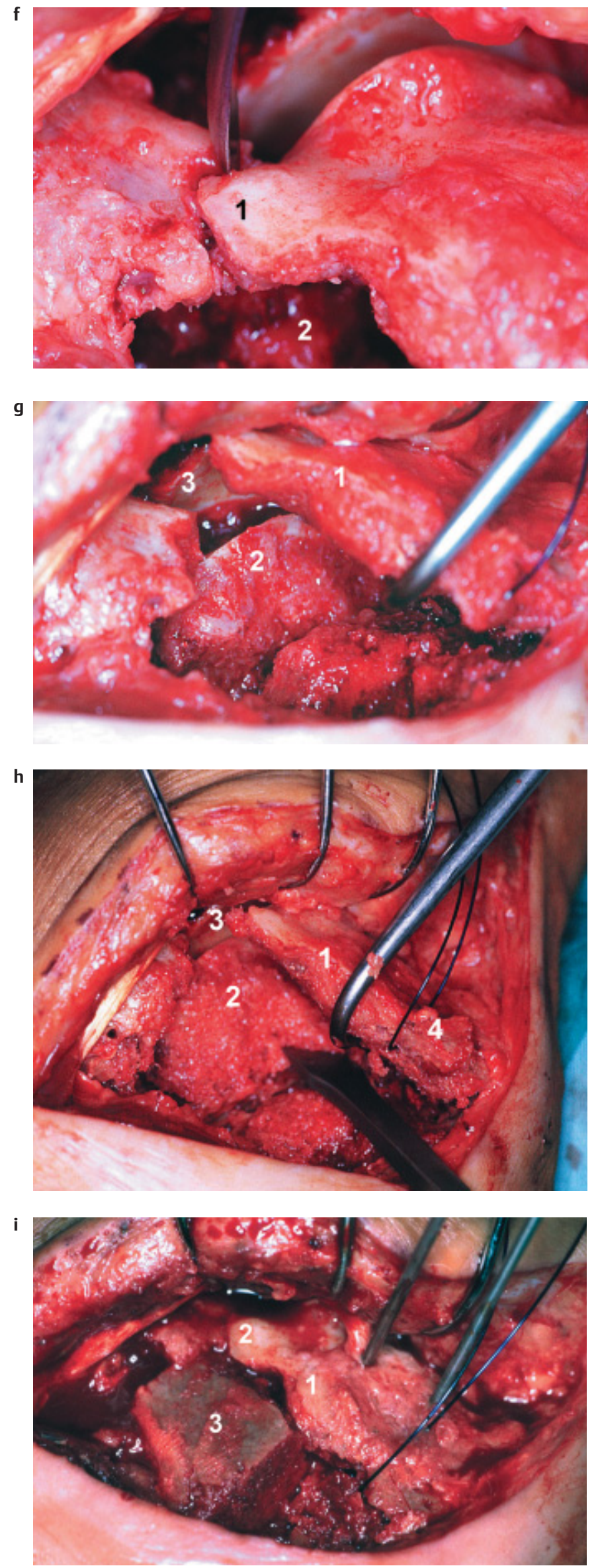
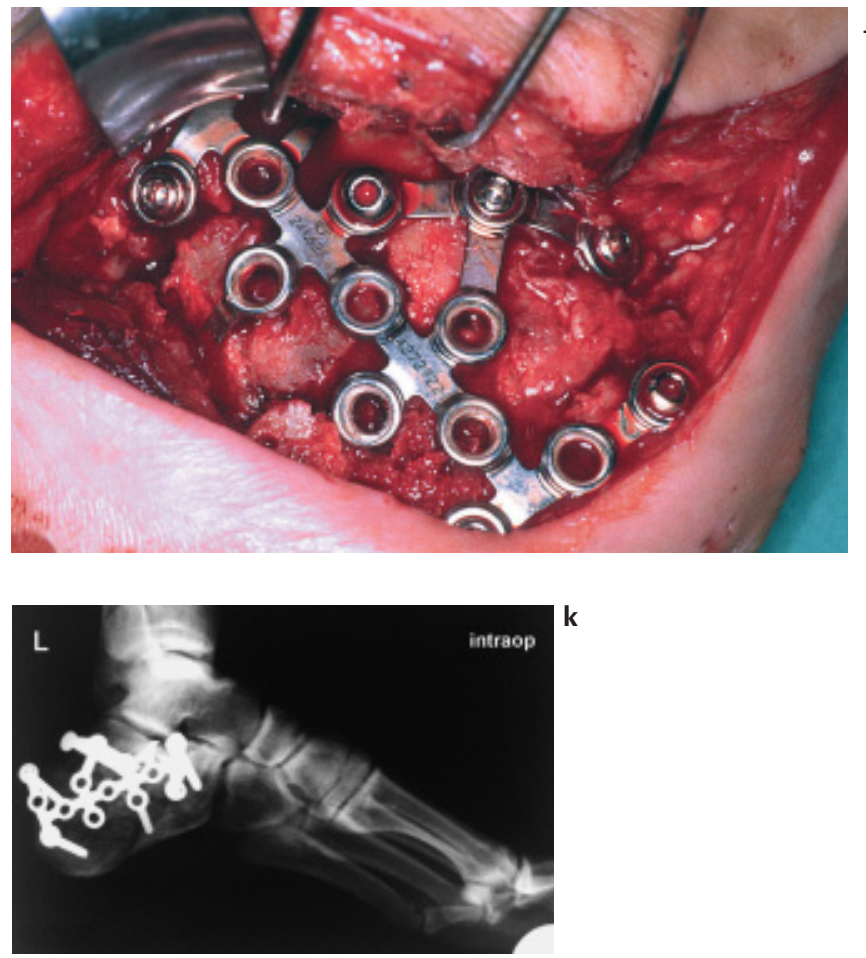

Abb. 2 Klinisches Fallbeispiel 1 einer frühen anatomischen Rekonstruktion 7 Wochen nach auswärtiger Calcaneus-Osteosynthese. (a) Bei dem 55-jährigen chirurgisch-tätigen Kollegen ist nach Osteosynthese der Böhler-Winkel scheinbar wiederhergestellt. Eine fragliche Doppelkontur und die fehlende Kongruenz zwischen posteriorer Facette des Calcaneus und des Talus mit nach kaudal sich öffnendem Winkel lässt eine Inkongruenz vermuten. (b, c) Ein nach Spickdrahtentfernung durchgeführtes $\mathrm{CT}$ ( 6 Wochen postoperativ) belegt mit den koronaren Schnitten eindeutig, dass die medialen Anteile der posterioren Facette nicht kongruent zum Talus ausgerichtet sind, so dass eine deutliche Verwerfung im Subtalar-Gelenk besteht. Das subthalamisch eingebrachte Endobone-Granulat (Hydroxylapatit) hat entweder nachgegeben oder die Fraktur war initial bereits nicht korrekt reponiert. Zusätzlich besteht eine mäßige Translation des Tubers nach lateral. (d) Auf Wunsch des Patienten wird ein früher Rekonstruktionsversuch mit der Option unternommen, bei Nichtrekonstruierbarkeit des Subtalar-Gelenkes alternativ eine reorientierende Subtalar-Arthrodese durchzuführen. Über den vorgegebenen ausgedehnt lateralen Zugang lässt sich das Subtalar-Gelenk nach Setzen von drei 2.0er K-Drähten in den gelenknahen Talus gut exponieren und imponiert initial nicht sehr auffällig. (e) Nach Entfernen des Plättchens erscheint der Calcaneus im Angle of Gissane (1) fest verheilt. Das subthalamisch eingebrachte Endobone-Granulat liegt völlig inert in einer großen Höhle (2). (f) Nach Ausräumen des Endobones stellt sich eine extrem große subthalamische Höhle (2) dar. Die Fraktur im Angle of Gissane (1) wird teils stumpf, teils mit dem Meißel gelöst. (g) Nach Osteotomie im Calcaneushals wird die laterale Wand (1) mit anhängendem Anteil der lateralen posterioren Facette mit einem Einzinkerhaken nach seitlich und oben weggehalten. Erst jetzt wird die erhebliche Fehlstellung des medialen Anteiles der posterioren Facette (2) gegenüber der Talusunterfläche (3) deutlich. (h) Nach Osteotomie gegenüber dem Tuber calcanei kann nun das medial gelenktragende Fragment (2) gegenüber dem Talus (3) reponiert und anatomisch mit einem temporären, von plantar eingebrachten 2.0er Spickdraht gehalten werden. Die laterale Wand mit dem lateralen Anteil der posterioren Facette (1) ist sicherheitshalber mit einer transossären Naht (4) zur Haut hin fixiert, um ein unverhofftes Abbrechen und Abstürzen zu vermeiden. (i) Die laterale Wand mit lateralem Anteil der posterioren Facette ist zum medialen Anteil der posterioren Facette und zum Talus hin jetzt anatomisch reponiert, ebenso zum Processus anterior calcanei im Angle of Gissane (2). Ein großer kortikospongiöser Span (3) ist in

(Fortsetzung auf Seite 239) 
(Fortsetzung von Seite 238)

den großen subthalamischen Defekt eingebracht. (j) Stabilisation aller gelösten Fragmente mit winkelstabiler Calcaneusplatte. (k) Die intraoperative Röntgenaufnahme zeigt im Vergleich zu a die jetzt anatomische Kongruenz des Subtalar-Gelenkes.

\section{b) Fehlstellung, Infekt, Weichteildefekt}

Weit schwieriger als die frühe Korrektur einer Fehlstellung ist das Management einer frühen Weichteil-Knocheninfektion bei liegendem Calcaneusplättchen. In diesen Fällen führt nur das radikale Dé-

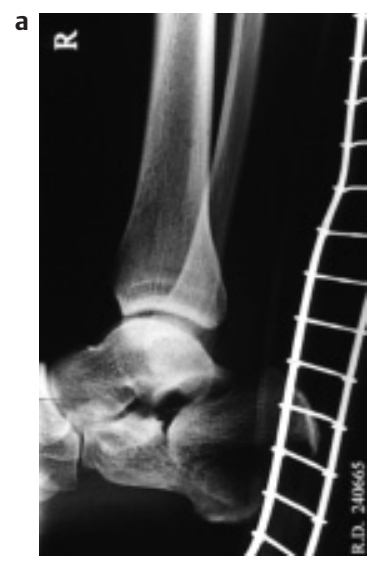

bridement von infizierten Weichteilen und nekrotischen Knochenanteilen unter Entfernung des Plättchens mit Reosteosynthese durch 3.5er Schrauben zur Minimierung des Fremdkörpers bei noch instabiler Fraktursituation zur sicheren Ausheilung. Problematisch ist gelegentlich die Weichteilbedeckung. Bei kleineren Defekten ist mit liegender Schraubenosteosynthese in der Regel eine Weichteilsanierung nach Konditionierung mit Meshgraft-Transplantation möglich. Größere Defekte der Fersenbeinweichteilummantelung sind nur durch eine freie mikrovaskuläre Lappenplastik beherrschbar. Das Fallbeispiel 2 (Abb. 3) zeigt, mit welch hohem Aufwand bei ausgedehnter Knochen- und Weichteilinfektion verbunden mit Weichteildefekt Lösungsmöglichkeiten gegeben sind [1].
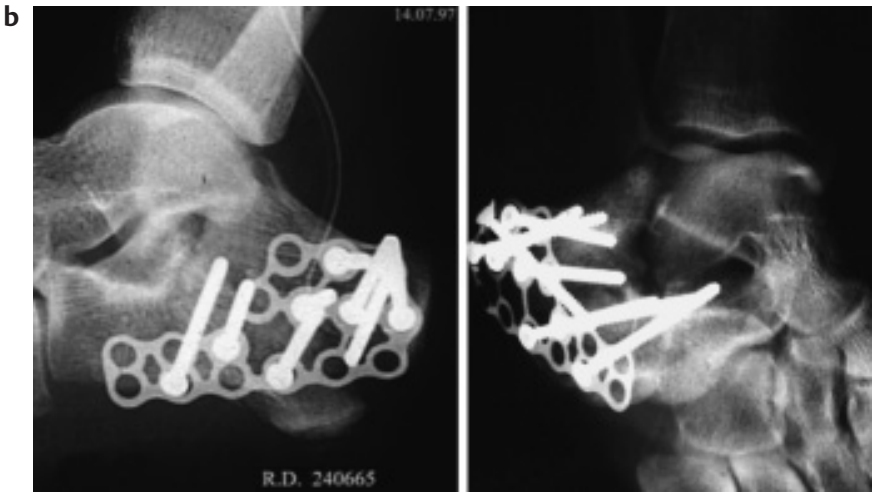

d

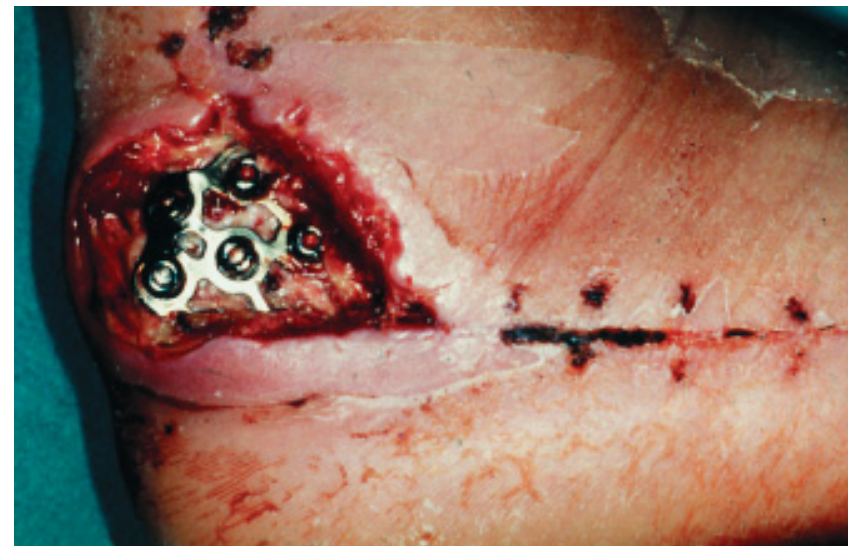

Abb. 3 Klinisches Fallbeispiel 2 für eine Fehlstellung, Weichteil- und Knocheninfektion mit großem Weichteildefekt. (a) Erheblich dislozierte „tongue type“-Fraktur bei einem 28-jährigen LKW-Fahrer nach Sturz von einer Rampe aus 2,5 m Höhe. Auswärtige Osteosynthese 5 Tage nach Trauma (b), wobei die nach Verlegung durchgeführte Brodén-Aufnahme (c) die persistierende Dislokation noch deutlicher zeigt. Bei Verlegung, 20 Tage nach Trauma, besteht ein kleinhandtellergroßer Hautdefekt über der freiliegenden Platte (d) mit putrider Sekretion aus der Tiefe. Nach Resektion des gesamten eitrig-nekrotischen Tuberfragmentes besteht ein riesiger Defekt mit abgelöstem Achillessehnenansatz
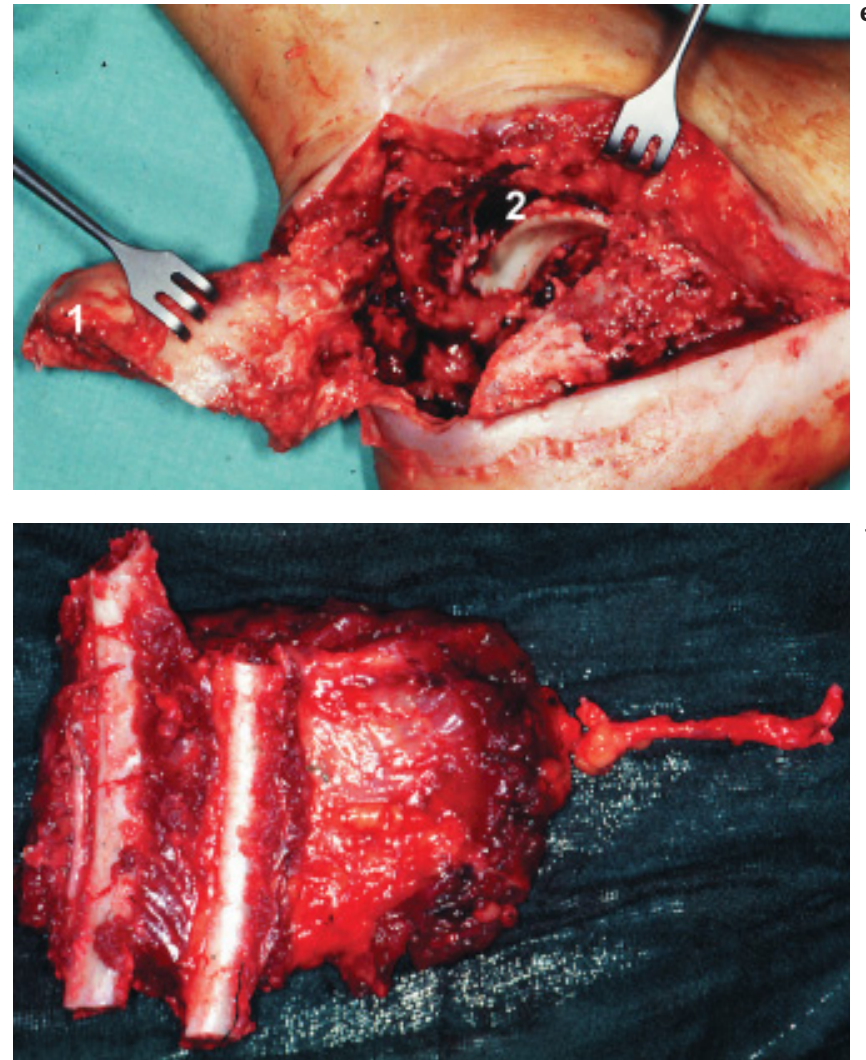

(1). Der Knorpel am Talusrand (2) ist etwas angedaut, aber unter dem Talus noch gut erhalten. Nach radikalem Débridement besteht eine subtotale Calcanektomie. (f) Nach Zweit-Débridement wird ein vaskularisierter Serratus-Lappen mit zwei gefäßversorgten Rippen gehoben, wobei durch den mikrovaskulär angeschlossenen Lappen einerseits die Rippen den großen knöchernen Defekt auffüllen und der dritte Muskelbauch den lateralen Wanddefekt seitlich und rückwärtig zur Achillessehne abdeckt, was in Teilabbildung (g, h, i) deutlich erkennbar ist. (i) Voll belastbarer Rückfuß 11/2 Jahre postoperativ. 
Abb. $3 \mathrm{~g}$
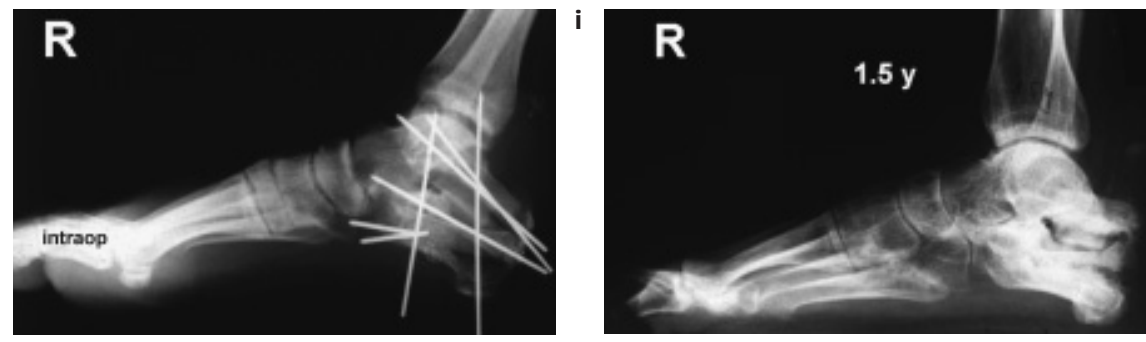

Abb. $3 \mathrm{~h}$
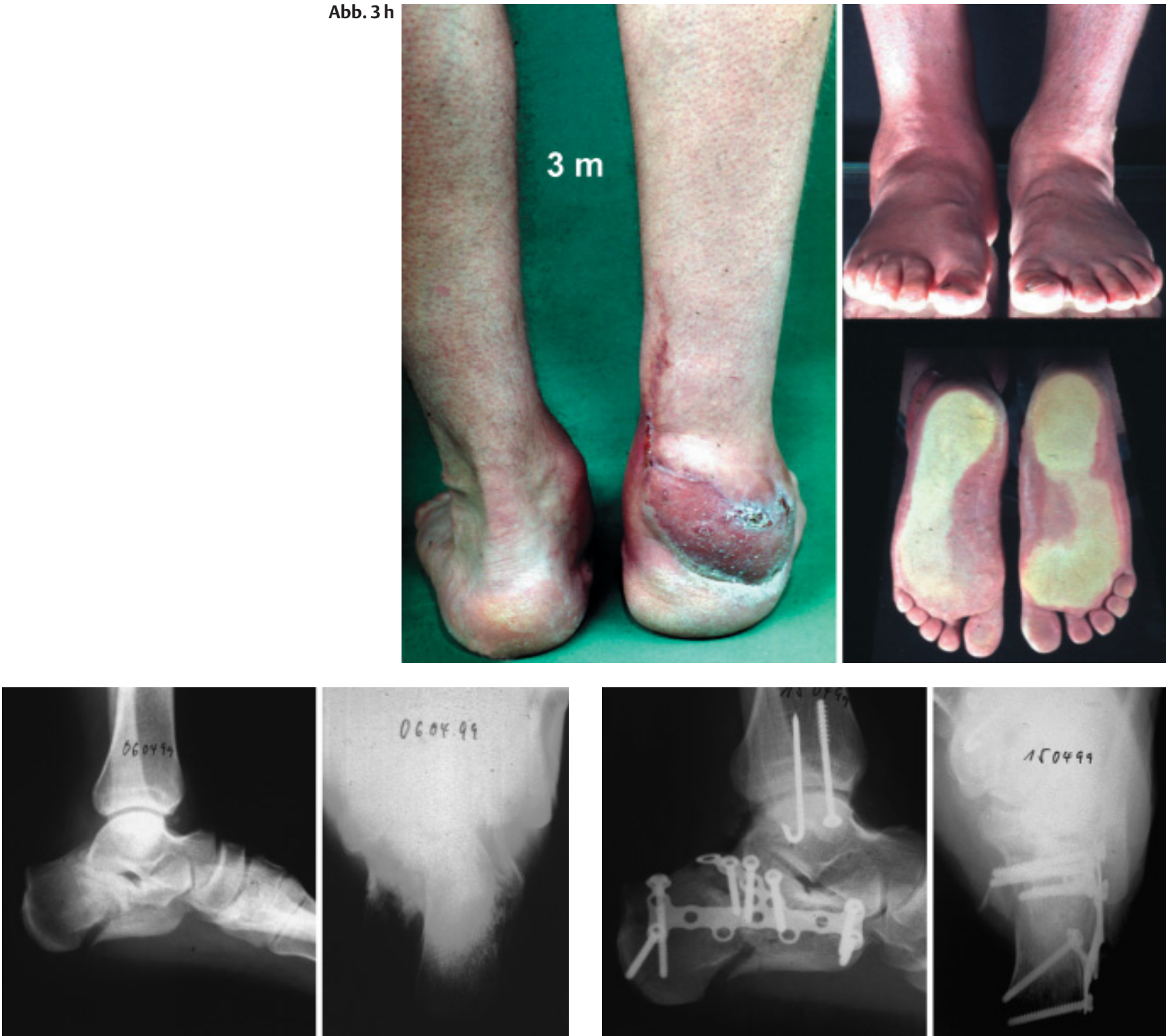

Abb. 4 Klinisches Fallbeispiel 3 einer Spätrekonstruktion: CalcaneusPseudarthrose nach fehlgeschlagener Calcaneus-Osteosynthese mit Kollaps des Rückfußes. (a) Die seitliche Rückfuß- und Axialaufnahme zeigen die 2-Gelenk-Fraktur des Calcaneus mit weitem Versatz des Tuberfragmentes nach lateral bei einem 62-jährigen Patienten. Ipsilaterale Innenknöchelfraktur. (b) Die seitliche Rückfuß- und Axialaufnahme zeigen, dass der Böhler-Winkel unmittelbar postoperativ nahezu anatomisch wiederhergestellt ist. Auch die Rückfußachse erscheint korrekt. Bei subtiler Analyse zeigen jedoch alle drei subthalamisch eingebrachten Schrauben zu weit nach plantar, so dass keine von ihnen das Sustentaculum fasst, was insbesondere die Axialaufnahme rechts verdeutlicht. (c) Aufgrund der Instabilität kommt es nach 4 Monaten zur Schraubenmigration, so dass diese bei lokalem Druck von innen auf die Haut in Lokalanästhesie entfernt wird. (d) Nach Entfernung des gesamten Implantates wird der
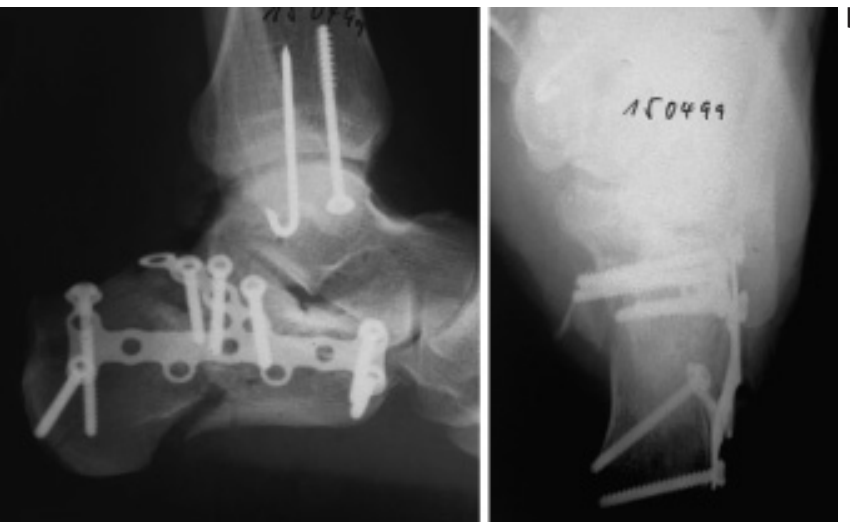

schwere Rückfußkollaps mit bestehender Pseudarthrose erst deutlich erkennbar. (e, f) Nach Konsolidierung der Weichteile wird 4 Wochen später die reorientierende subtalare Arthrodese durchgeführt, wobei mit der eingebrachten Schanz-Schraube das Tuber nach plantar gehebelt wird (1). Über einen posterolateralen Zugang wird der Restknorpel aus dem Subtalar-Gelenk mit scharfem Löffel entfernt (2) und ausreichend große Späne von (3) dorsal eingebracht. Interposition von freier autologer Spongiosa zwischen Tuber und Processus anterior. Kompression auf das Pseudarthrosenareal mit einer 6.5er Spongiosazugschraube (4). Stabilisierung des Rückfußes mit Kompression auf die subthalamisch eingebrachten autologen kortikospongiösen Blöcke durch zwei 6.5er Spongiosazugschrauben (5). (g, h) Die postoperativen Verlaufskontrollen 3 und 10 Monate postoperativ zeigen die rasche knöcherne Heilung und anatomische Reorientierung des Rückfußes bei völliger Beschwerdefreiheit des Patienten. 


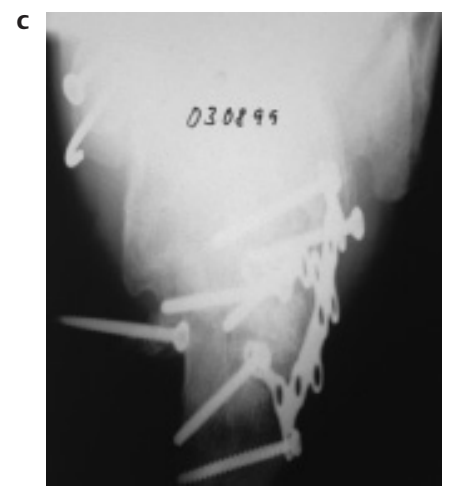

Abb. 4

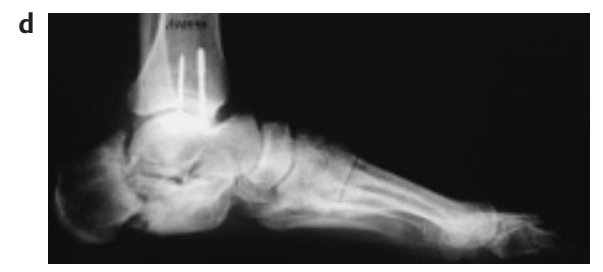

a
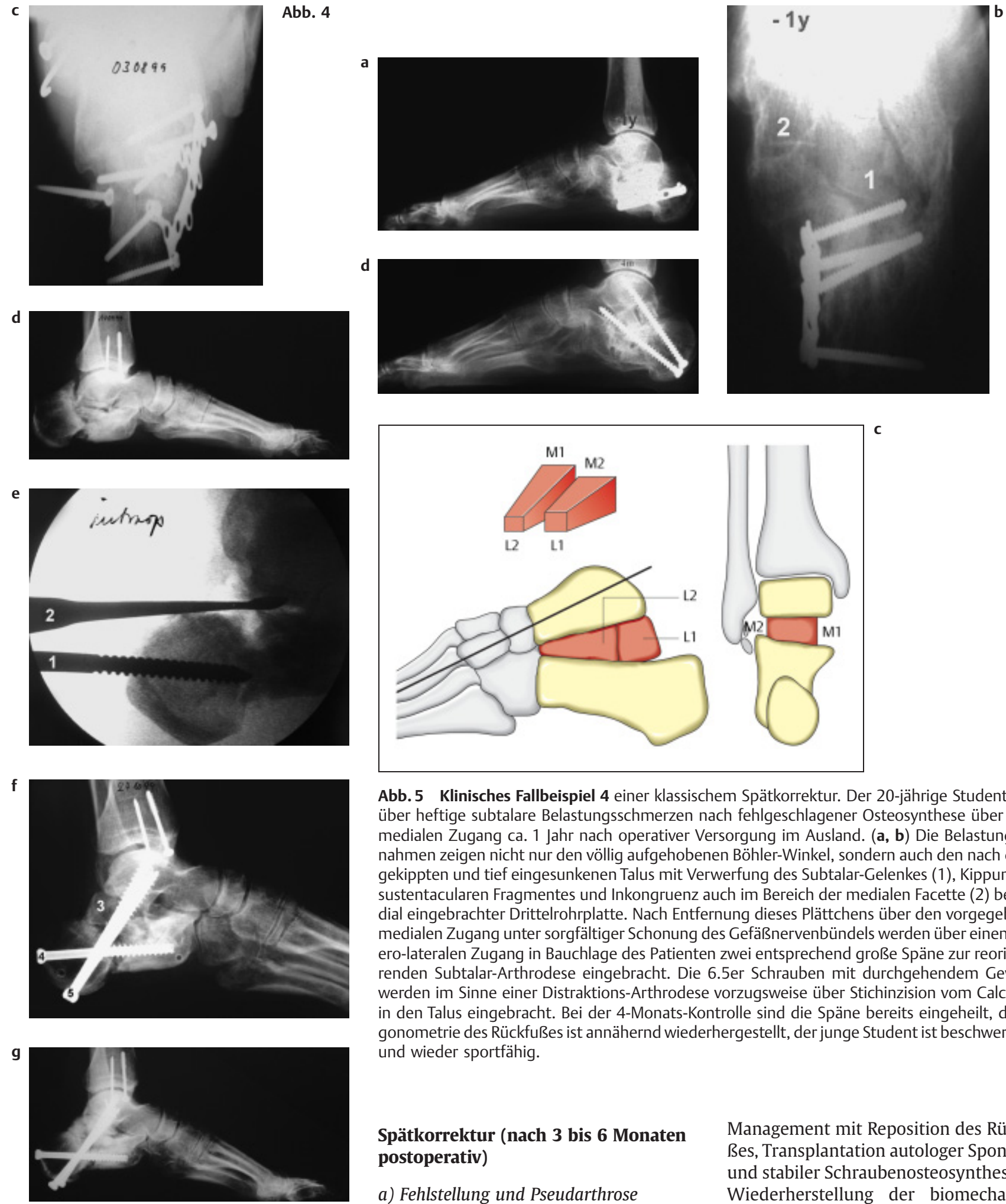

Abb. 5 Klinisches Fallbeispiel 4 einer klassischem Spätkorrektur. Der 20-jährige Student klagt über heftige subtalare Belastungsschmerzen nach fehlgeschlagener Osteosynthese über einen medialen Zugang ca. 1 Jahr nach operativer Versorgung im Ausland. (a, b) Die Belastungsaufnahmen zeigen nicht nur den völlig aufgehobenen Böhler-Winkel, sondern auch den nach dorsal gekippten und tief eingesunkenen Talus mit Verwerfung des Subtalar-Gelenkes (1), Kippung des sustentacularen Fragmentes und Inkongruenz auch im Bereich der medialen Facette (2) bei medial eingebrachter Drittelrohrplatte. Nach Entfernung dieses Plättchens über den vorgegebenen medialen Zugang unter sorgfältiger Schonung des Gefäßnervenbündels werden über einen postero-lateralen Zugang in Bauchlage des Patienten zwei entsprechend große Späne zur reorientierenden Subtalar-Arthrodese eingebracht. Die 6.5er Schrauben mit durchgehendem Gewinde werden im Sinne einer Distraktions-Arthrodese vorzugsweise über Stichinzision vom Calcaneus in den Talus eingebracht. Bei der 4-Monats-Kontrolle sind die Späne bereits eingeheilt, die Trigonometrie des Rückfußes ist annähernd wiederhergestellt, der junge Student ist beschwerdefrei und wieder sportfähig.

\section{Spätkorrektur (nach 3 bis 6 Monaten postoperativ)}

\section{a) Fehlstellung und Pseudarthrose}

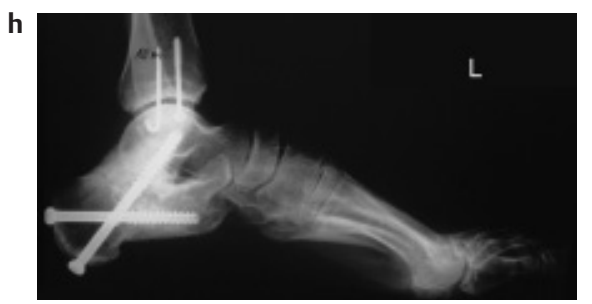

Wenngleich Pseudarthrosen mit oder ohne Kollaps des Rückfußes nach operativ versorgten Calcaneusfrakturen extrem selten sind, d.h. im eigenen Krankengut in 2 von 453 kontrollierten Fällen [6], muss dennoch ein klares Konzept zur Korrekturlösung vorhanden sein. Das Fallbeispiel 3 (Abb.4) soll das operative
Management mit Reposition des Rückfußes, Transplantation autologer Spongiosa und stabiler Schraubenosteosynthese zur Wiederherstellung der biomechanisch wichtigen Trigonometrie des Fußes illustrieren.

b) Fehlstellung und Arthrose des SubtalarGelenkes

Die am häufigsten angewandte Variante von Korrekturmöglichkeiten fehlgeschlagener Osteosynthesen am Fersenbein ist 
die risikoärmste und funktionell einschneidenste, da sie immer mit einer Arthrodese des Subtalar-Gelenkes verbunden ist. Ist es durch primäres oder sekundäres Osteosyntheseversagen zum postoperativen Kollaps des Rückfußes gekommen, erscheint es zweckmäßig, die Korrektur in zwei Schritten vorzunehmen. Im ersten Schritt sollte das in der Regel lateral liegende Implantat entfernt werden, um dann im zweiten Schritt ca. 3 bis 6 Monate später über einen posterolateralen Zugang den Restknorpel aus dem Subtalar-Gelenk zu entfernen, den Rückfuß aufzurichten und mit kortikospongiösen Spänen und Schrauben zu stabilisieren. Durch diesen posterolateralen Zugang ist sichergestellt, dass bei Kollaps des Rückfußes mit Schrumpfung der Haut und neuerlicher Aufrichtung des Rückfußes kein Weichteilproblem entsteht. Neu bei geringem oder fehlendem Rückfußkollaps kann die Implantatentfernung über den ausgedehnt lateralen Zugang mit anschließender Arthrodese in einem einzeitigen Vorgehen empfohlen werden. Die Prinzipien der reorientierenden Subtalar-Arthrodese sind in Abb. 5 beispielhaft dargestellt.

\section{Zusammenfassung}

Lösungsmöglichkeiten nach fehlgeschlagenen Osteosynthesen am Calcaneus sind begrenzt. Insbesondere beinhaltet die Frühkorrektur, d.h. innerhalb 3 bis 6 Wochen postoperativ, ein deutlich erhöhtes Risiko für den Weichteilverschluss, die Weichteil- und Knochenheilung. Sie ist operationstechnisch extrem anspruchsvoll, da eine rasche Kallusentwicklung eventuell Osteotomien notwendig macht, der Knochen zur Restabilisierung bei inaktivitätsosteoporotischer Weichheit schlecht zu refixieren ist und die Weichteile in der Regel äußerst fragil sind. Spätkorrekturen, d.h. Lösungsmöglichkeiten nach 3 bis 6 Monaten postoperativ, haben grundsätzlich ein wesentlich geringeres Operationsrisiko, sind technisch einfacher als Frühkorrekturen, haben aber prinzipiell den Nachteil, dass sie bei verworfenen Gelenkfrakturen nicht mehr gelenkerhaltend, sondern immer im Sinne der reorientierenden Subtalar-Arthrodese auszuführen sind.

\section{Literatur}

1 Brenner P, Zwipp H, Rammelt S. Vascularized Double Barrels Ribs Combined with Free Serratus Anterior Muscle Transfer for Homologous Restoration of the Hindfoot after Calcanectomy. J Trauma 2000; 49: 331 335

2 Gavlik JM, Rammelt S, Barthel S, Zwipp H. Operationsmöglichkeiten und Indikation bei Fersenbeinfrakturen. Chir Praxis 2001; 58: 649-664

3 Rammelt S, Barthel S, Biewener A, Gavlik JM, Zwipp H. Calcaneusfrakturen - offene Reposition und interne Stabilisierung. Zentralbl Chir 2003; 128: 517-528

4 Wentzensen A. Intraoperative 3D-Darstellung am Calcaneus. 2. Deutscher AO-Fußkurs 07.10. - 10.10.2003, Dresden

5 Zwipp H, Rammelt S, Barthel S. Winkelsteife Implantate am Fersenbein, warum?. OPJournal 2004; 20: 32 - 36

6 Zwipp H, Rammelt S, Barthel S. Calcaneal fractures: ORIF. Injury Suppl 200435 $2: 46-54$

Prof. Dr. med. Hans Zwipp

Klinikdirektor

Dr. med. Stefan Rammelt

Funktionsoberarzt

Dr. med. René Grass

Leitender Oberarzt

Univ.-Klinikum Carl Gustav Carus

der TU Dresden

Klinik und Poliklinik für Unfall- und

Wiederherstellungschirurgie

Fetscherstr. 74

D-01307 Dresden 